\title{
Modeling neutrino-nucleus interactions in the few-GeV region
}

\author{
Kajetan Niewczas* \\ Department of Physics and Astronomy, Ghent University, Gent, Belgium \\ Institute of Theoretical Physics, University of Wroctaw, Wroctaw, Poland \\ E-mail: Kajetan.Niewczas@uGent. be
}

Natalie Jachowicz, Alexis Nikolakopoulos, Jannes Nys, Nils Van Dessel

Department of Physics and Astronomy, Ghent University, Gent, Belgium

\section{Raul González-Jiménez}

Department of Atomic, Molecular and Nuclear Physics, Complutense University of Madrid, Madrid, Spain

\section{Vishvas Pandey}

Center for Neutrino Physics, Virginia Tech, Blacksburg, Virginia, USA

\begin{abstract}
A good understanding of neutrino-nucleus scattering mechanisms is essential to reduce the systematic errors in neutrino oscillation experiments. The Ghent group focuses on providing a consistent description of this process in the intermediate energy region. We describe the low energy response with collective nuclear excitations and the quasielastic peak using a Hartree-FockCRPA (continuum random phase approximation) model that takes into account nuclear longrange correlations as well as hadronic final-state interactions. The two-body current mechanisms, which are especially important in the region between the quasielastic and the delta-resonance peak, are included through short-range correlations and meson-exchange currents, treated within the same mean-field based model. Our description of intermediate-energy neutrino-nucleus scattering is completed by modeling neutrino-induced pion production. For that, we consider the dominant contribution from the decay of the delta and higher-mass resonances as well as other terms required by chiral symmetry, working in a fully relativistic formalism with a refined treatment of nuclear effects.
\end{abstract}

The 20th International Workshop on Neutrinos (NuFact2018)

12-18 August 2018

Blacksburg, Virginia

${ }^{*}$ Speaker. 


\section{Introduction}

The precise description of neutrino-nucleus interactions is an essential weapon in the quest to reduce systematical uncertainties in neutrino oscillation experiments [1]. Most of the present generation experiments are exploring the intermediate energy region, where the lepton emitted in the charged-current (CC) reaction is accompained by a hadronic final state with one (1p1h) or two (2p2h) nucleons, and a possible pion (SPP) leaving the residual nucleus. The goal of the Ghent group is to describe all of the relevant processes within a consistent, theoretically well-motivated framework, using a mean-field based model for both the initial and final hadronic states.

\section{Models and Results}

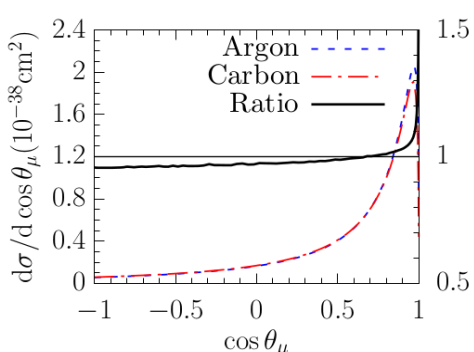

(a) 1p1h: MicroBooNE fluxfolded $\mathrm{CC}$ single-differential cross section.

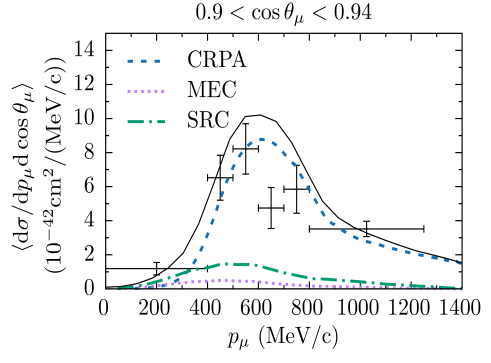

(b) $2 \mathrm{p} 2 \mathrm{~h}: \mathrm{T} 2 \mathrm{~K}$ flux-folded $\mathrm{CC} 0 \pi$ double-differential cross section and data [12].

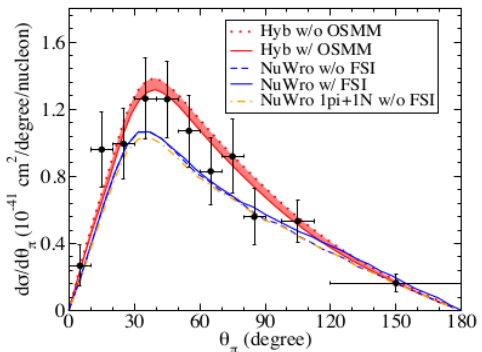

(c) SPP: MINERvA flux-folded $\mathrm{CC} 1 \pi^{+}$single-differential cross section and data [13].

Figure 1: Predictions of the models developed by the Ghent group, adapted from Ref. [2, 4, 7].

\subsection{Giant Resonances region and Quasi-elastic peak}

The wave functions of bound nucleons are calculated solving the Schrödinger equation with a self-consistent mean-field potential generated by an effective Skyrme nucleon-nucleon interaction, following a Hartree-Fock (HF) procedure. Long-range correlations are introduced within a continuum random phase approximation (CRPA) that allows for a collective nucleon treatment. The outgoing nucleon wave functions are under influence of the same nuclear potential, accounting for elastic final-state interactions (FSI). Fig. 1a presents the ratio of Argon to Carbon cross sections [2], relevant for LArTPC experiments, such as MicroBooNE.

\subsection{Two-nucleon knockout: SRC and MEC}

Extending the model described in the previous section, the reactions leading to the knockout of two nucleons are modeled here in terms of short-range correlations (SRC) and meson-exchange currents (MEC). The former are the effects than can be attributed to the properties of the ground state nuclei and are introduced using a correlation operator $\hat{G}$ that is later contained within an effective operator $\hat{J}_{\text {eff }} \sim \hat{G}^{\dagger} \hat{J} \hat{G}$ [3]. The latter explicitly include contribution from Feynam diagrams describing two-body currents [4]. The results presented in Fig. 1b show the significance of the $2 \mathrm{p} 2 \mathrm{~h}$ final states contribution for the inclusive neutrino-nucleus cross sections. The contribution 
from other interaction channels, that supply this signal through inelastic FSI, are not present in the model [5].

\subsection{Single-pion production}

The description of single-pion production channels is done within the Hybrid-RPWIA model presented in Ref. [6, 7, 8]. The low-energy base for this model is the model of Ref. [9], that includes both the resonance pion production and background contributions (non-resonant pion production), while the usage of a Regge approach extends the model into scattering at high energies. This single-pion production off nucleon model is embedded into nuclear medium using the framework of the Relativistic Mean-Field (RMF) for the calculation of nucleon wave functions. The inmedium modifications of the $\Delta$-resonance (OSMM) is estimated according to the prescription of Ref. [10]. In Fig. 1c, the results of the Hybrid-RPWIA model have been succesfully confronted with the MINERvA CC1 $\pi^{+}$data. Here, the FSI effect was estimated using the predictions of the Monte Carlo neutrino event generator NuWro [11].

\section{Summary}

The Ghent group is actively developing models allowing for consistent predictions of neutrinonucleus cross sections in the energy range covered by the accelerator-based neutrino oscillations experiments. The work is ongoing to include: FSI effects for single-pion production, $\Delta$-currents in two-nucleon knockout, and to perform basic implementations of the models in the NuWro event generator.

\section{Acknowledgments}

This work was supported by the Research Foundation Flanders (FWO-Flanders), and the Special Research Fund, Ghent University. The computational resources and services used in this work were provided by Ghent University, the Hercules Foundation and the Flemish Government. K.N. was partially supported by the Polish National Science Center (NCN), under Opus Grant No. 2016/21/B/ST2/01092. R.G.J. was supported by Comunidad de Madrid and UCM under the contract No. 2017-T2/TIC-5252. V.P. acknowledges the support by the National Science Foundation under Grant No. PHY-1352106.

\section{References}

[1] L. Alvarez-Ruso et al. (NuSTEC Collaboration), Prog.Part.Nucl.Phys. 100 (2018) 1-68

[2] N. Van Dessel et al., Phys.Rev. C97 (2018) 044616

[3] T. Van Cuyck et al., Phys.Rev. C94 (2016) 024611

[4] T. Van Cuyck et al., Phys.Rev. $\mathbf{C 9 5}$ (2017) 054611

[5] K. Niewczas et al., Springer Proc. Phys. 225 (2019) 155-156

[6] R. González-Jiménez et al., Phys.Rev. D95 (2017) 113007

[7] R. González-Jiménez et al., Phys.Rev. D97 (2018) 013004 
[8] A. Nikolakopoulos et al., Phys.Rev. D97 (2018) 093008

[9] E. Hernández et al., Phys.Rev. D76 (2007) 033005

[10] E. Oset and L.L. Salcedo., Nucl. Phys. A468 (1987) 631-652

[11] T. Golan et al., Phys.Rev. C86 (2012) 015505

[12] K. Abe et al. (T2K Collaboration), Phys.Rev. D93 (2016) 112012

[13] B. Eberly et al. (MINERvA Collaboration), Phys.Rev. D92 (2015) 092008 